\title{
Optimal insurance contract under a value-at-risk constraint
}

\author{
Hung-Hsi Huang
}

Received: 30 August 2005 / Revised: 30 March 2006

(C) Springer Science + Business Media, LLC 2006

\begin{abstract}
This study develops an optimal insurance contract endogenously under a value-at-risk (VaR) constraint. Although Wang et al. [2005] had examined this problem, their assumption implied that the insured is risk neutral. Consequently, this study extends Wang et al. [2005] and further considers a more realistic situation where the insured is risk averse. The study derives the optimal insurance contract as a single deductible insurance when the VaR constraint is redundant or as a double deductible insurance when the VaR constraint is binding. Finally, this study discusses the optimal coverage level from common forms of insurances, including deductible insurance, upper-limit insurance, and proportional coinsurance.
\end{abstract}

Keywords Value-at-risk $\cdot$ Optimal insurance $\cdot$ Deductible $\cdot$ Policy limit · Coinsurance

\section{JEL Classification G22}

\section{Introduction}

This study aims to develop an optimal insurance contract endogenously under a valueat-risk (VaR) constraint, where VaR is defined as the worst expected loss over a given horizon at a particular confidence level [Jorion, 2001]. Although Wang et al. [2005] had examined this problem, their assumption implied that the insured is risk neutral. Consequently, this study extends Wang et al. [2005] and further considers a more realistic situation where the insured is risk averse. ${ }^{1}$

\footnotetext{
H.-H. Huang $(\bowtie)$

Department of Business Administration, Southern Taiwan University of Technology, No. 1, Nan-Tai Street, Yung-Kang, Taiwan e-mail: d86723002@ntu.edu.tw

${ }^{1}$ The reasons why VaR is considered in the optimal insurance contract problem had been presented in Wang et al. [2005]. Since this paper is a mere extension of Wang et al. [2005], these reasons are dropped here.
} 
Previous studies on insurance demand primarily include two classes. The first class only attempts to determine the optimal coverage, given that the insurance contracts have the common forms (including deductible insurance, proportional coinsurance, and upper-limit policy), for example: Schlesinger [1981], Doherty and Schlesinger [1983a,b], and Huberman et al. [1983]. Meanwhile, the second class attempts to identify the optimal insurance contract endogenously, for example: Raviv [1979], Gollier [1987, 1996], Spaeter and Roger [1997], Ermoliev and Flam [2001], and Wang et al. [2005]. Actually, the solution processes for the insurance demand in the two classes are not the same. The first class solution only requires one stage and may be solved by fundamental calculus, while the second class requires two stages. Meanwhile, the first stage in the second class frequently uses variation of calculus to find the optimal indemnity schedule given a fixed premium. The second stage then uses the fundamental calculus to yield the optimal premium.

If the insurance contract must meet the VaR constraint, the first stage in the second class cannot use the standard variation of calculus to find the optimal indemnity schedule (see Section 2). Accordingly, Wang et al. [2005] adopted a logical analysis approach and directly derived an optimal indemnity schedule. Although the VaR constraint in this study is similar to that used by Wang et al. [2005], our objective function is to maximize expected utility rather than expected wealth in Wang et al. [2005]. Due to the added complexity for utility specification, the optimal indemnity schedule presented in this study cannot be obtained by the same approach as that in Wang et al. [2005]. Instead, first a potential candidate is selected among all feasible indemnity schedules. This candidate is identified as the optimal indemnity schedule, if the candidate is not dominated by all the others. The results demonstrate that the optimal insurance is single deductible insurance when the VaR constraint is redundant or double deductible insurance when the VaR constraint is binding (see Section 3).

The remainder of this paper is organized as follows. Section 2 introduces the assumptions and the optimality problem of the insured. Next, Section 3 derives the optimal retained loss schedule. Section 4 then describes the selection about insurance premiums and contractual forms, since insurance premiums and contractual forms have mutual effects on one another. Subsequently, Section 5 discusses the optimal coverage from the commonly existing forms of insurance, including deductible insurance, upper-limit insurance, and proportional coinsurance. Finally, conclusions are presented in Section 6.

\section{The model}

Similar to Raviv [1979], Gollier [1996], and Wang et al. [2005], this study makes the following assumptions. An insured with initial wealth $W_{0}$ faces a risk of loss $X$, where $X$ is a continuous random variable with probability density function $f(x)$ and cumulative distribution function $F(x) . X$ is non-negative and no more than the upper bound $T$, hence its sample space $S=\{x \mid 0 \leq x \leq T\}$. The insured has the opportunity to purchase insurance which costs a premium $P$ and pays an indemnity schedule $I(x)$, 
$0 \leq I(x) \leq x$ for all $x$. Additionally, the premium $P$ is assumed to be as follows. ${ }^{2}$

$$
P=C(\bar{I}), \quad C(0)=0, \quad \text { and } \quad C^{\prime}(\bar{I})>1, \quad C^{\prime \prime}(\bar{I})>0 \quad \text { for all } \bar{I}>0
$$

where $\bar{I}=E[I(X)]$ denotes the expected indemnity, $C^{\prime}(\bar{I})>1$ represents that the marginal premium exceeds the marginal expected indemnity, and $C^{\prime \prime}(\bar{I})>0$ indicates that the insurer is likely to be risk averse. Accordingly, the final and expected final wealth of the insured are $W=W_{0}-P-X+I(X)$ and $\bar{W}=W_{0}-P-\bar{x}+\bar{I}$ respectively, where $\bar{x}=E[X]$ denotes the expected loss.

For regulation or risk management, sometimes $W$ must meet the VaR constraint. As defined in Section 1 , the $\operatorname{VaR}$ constraint means that $\operatorname{Pr}\{W \geq \bar{W}-v\} \geq 1-\alpha$, where $v \equiv \mathrm{VaR}$ and $1-\alpha$ denotes the confidence level. The insured is risk averse with a utility function $U(W), U^{\prime}(\cdot)>0>U^{\prime \prime}(\cdot)$. Following Raviv [1979] and Gollier [1996], the objective of the insured is to choose a couple $(P, I(x))$ to maximize the expected final wealth utility $E[U(W)]$ under the VaR constraint. Compared with $I(x)$, the retained loss schedule $R(x) \equiv x-I(x)$ is more convenient for deriving the result in this study, and thus the couple is changed to $(P, R(x))$. Since $W=$ $W_{0}-P-X+I(X)$, the $\operatorname{VaR}$ constraint $\operatorname{Pr}\{W \geq \bar{W}-v\} \geq 1-\alpha$ is equivalent to

$$
\operatorname{Pr}\{X-I(X) \leq v+\bar{x}-\bar{I}\} \geq 1-\alpha
$$

Consequently, the optimality problem of the insured is as follows.

$$
\underset{P \geq 0,0 \leq R(x) \leq x}{\operatorname{Maximize}} E[U(W)]=E\left[U\left(W_{0}-P-R(X)\right)\right]
$$

subject to

$$
\begin{aligned}
P= & C(\bar{I}), \quad C(0)=0, \quad C^{\prime}(\bar{I})>1, \quad C^{\prime \prime}(\bar{I})>0 \\
\bar{I}= & E[I(X)]=E[X-R(X)] \\
& \operatorname{Pr}\{R(X) \leq K\} \geq 1-\alpha, \quad K \equiv v+\bar{x}-\bar{I}
\end{aligned}
$$

Using integral representations, expression (3) likely appears to be a continuous optimal control problem, which is frequently solved using the Lagrangean approach, Hamiltonian approach, and the variations of calculus [Fryer and Greenman, 1987]. However, owing to the VaR constraint in expression (3c), expression (3) cannot form

\footnotetext{
${ }^{2}$ The premium setting in this study resembles that of Gollier [1996]; that is, the premium is based upon the expected indemnity: $P=C(E[I(X)])$. Notably, a number of studies simply assume $P=$ $(1+l)(E[I(X)]), 0<l<1$ is constant; for example, Doherty and Schlesinger [1983a,b] and Wang et al. [2005].
} 
a standard control problem. ${ }^{3}$ Consequently these traditional methods are difficulty to apply, and a new approach must be developed for solving the optimality problem of the insured.

Define $A$ is the $100(1-\alpha)$ th percentile of $X, F(A)=1-\alpha$ or $A=F^{-1}(1-\alpha)$, where $F^{-1}(\cdot)$ denotes the inverse function of $F(\cdot)$ and analogous representations are contained throughout this study. For convenience, this study additionally assumes

Both $\alpha$ and $v$ are small enough such that $v+\bar{x}<A$

In practice, $\alpha$ is frequently set to $5 \%$ or less and $v$ is small compared with the expected loss. Hence, the relation $v+\bar{x}<A$ meets the most practical situation.

\section{Optimal retained loss schedule}

As in Raviv [1979], Gollier [1987, 1996], and Wang et al. [2005], expression (3) is solved via two steps. First, the premium $P$ is assumed to be fixed and the optimal retained loss schedule $R(x)$ is found as a function of $P$. Second, the optimal $P$ and the corresponding insurance contract are selected. To describe the main result, the single deductible insurance and the double deductible insurance is defined as follows. Additionally, a preliminary result of this study is obtained in Theorem 1 below.

Definition 1. A single deductible insurance indicates that the insurance has a single deductible $D$ such that the retained schedule $R(x)=\min \{x, D\}$ for all loss $x$.

Definition 2. A double deductible insurance indicates that the insurance contains two deductibles, a lower deductible $\underline{D}$, a upper deductible $\bar{D}$, and a threshold $\hat{x}$ such that the retained schedule $R(x)=\min \{x, \underline{D}\}$ for losses $x \leq \hat{x}$ and $R(x)=\min \{x, \bar{D}\}$ for losses $x>\hat{x}$.

Theorem 1. If the VaR constraint is redundant, the optimal insurance forms a single deductible insurance. Restated, the optimal retained schedule

$$
R^{*}(x)= \begin{cases}x & \text { for } 0 \leq x<D^{*} \\ D^{*} & \text { for } D^{*} \leq x \leq T\end{cases}
$$

\footnotetext{
${ }^{3}$ If there is no VaR constraint and simply $P=C(\bar{I})=(1+l) \bar{I}, 0<l<1$ is constant, then this problem forms a standard optimal control problem and can be solved by maximizing the Hamiltonian, as follows.

$$
\underset{R(x), \lambda}{\operatorname{maximize}} H=\left\{U\left(W_{0}-P-R(x)\right\}+\lambda\{P-(1+l)[x-R(x)]\} f(x) \text { for all } x\right.
$$

where $\lambda$ is constant with respecte to $x$. However, if this problem adds a VaR restriction of expression (3c), then the Hamiltonian cannot be represented and expression (3) cannot form a standard optimal control problem. Additionaly, even though no VaR constraint exists, if $C(\bar{I})$ is not linear in $\bar{I}$ then the Hamiltonian also cannot be found. The related discussions are presented in Fryer and Greenman [1987], Raviv [1979], and Spaeter and Roger [1997].
} 
Fig. 1 Single deductible insurance

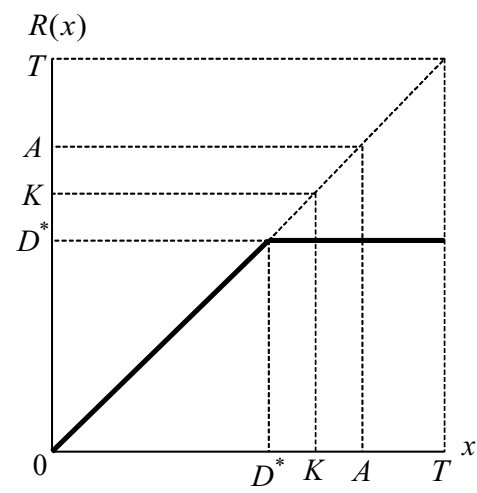

where $D^{*}$ represents the optimal deductible such that

$$
\int_{0}^{D^{*}} x f(x) d x+D^{*} \int_{D^{*}}^{T} f(x) d x=E[R(X)]=\bar{x}-\bar{I}
$$

The proof process of Theorem 1 is analogous to that presented by Bowers et al. [Chapter 1, 1986]. Actually, a single deductible insurance is commonly called a deducible insurance in Raviv [1979] and the traditional insurance literature. When the VaR constraint is released, the optimality problem of the insured is reduced to the model in Raviv [1979] and thus a single deductible insurance would be optimal. Although the result of Theorem 1 is not surprising, the condition that the VaR constraint is redundant is interesting and hence is discussed below. Based on expressions (3c) and (4), we obtain the relation $D^{*} \leq K \leq A$. Accordingly, the single deductible insurance would resemble the graph in Fig. 1.

This study now discusses the second case in which the VaR constraint is binding and thus influences the selection of $R(x)$.

Theorem 2. Assume that $S$ is the sample space of random variable $X ; S_{1}$ and $S_{2}$ partition $S: S_{1} \cup S_{2}=S$ and $S_{1} \cap S_{2}=\phi ; R(x)$ denotes the retained loss and

$$
R(x)= \begin{cases}R_{1}(x) & \text { for } x \in S_{1} \\ R_{2}(x) & \text { for } x \in S_{2}\end{cases}
$$

Constraining $R_{1}(x)=R_{1}^{c}(x)$ for $x \in S_{1}$ and $E[R(X)]=\bar{R}$, then the optimal retained loss $R_{2}(x)=R_{2}^{*}(x)$ takes the form

$$
R_{2}^{*}(x)= \begin{cases}x & \text { for } x<D^{*}, x \in S_{2} \\ D^{*} & \text { for } x \geq D^{*}, x \in S_{2}\end{cases}
$$


where the deductible $D^{*}$ is the solution of

$$
\int_{\substack{x<D^{*} \\ x \in S_{2}}} x f(x) d x+D^{*} \int_{\substack{x \geq D^{*} \\ x \in S_{2}}} f(x) d x=\bar{R}-\int_{x \in S_{1}} R_{1}^{c}(x) f(x) d x
$$

Proof: See Appendix 1.

Without further constraints, Theorem 1 shows that the Pareto optimal insurance takes the form of a single deductible insurance. When a risk has been insured against a specific loss interval, Theorem 2 shows that the optimal supplement insurance is also a form of single deductible insurance for covering the remaining loss interval.

To obtain Theorem 3 below, this study first considers Fig. 2, in which the corresponding premium is $P=P_{\min }$ on Panel A, $P_{\min }<P<P_{A}$ on Panel B, $P=P_{A}$ on Panel C, and $P_{A}<P<P_{K}$ on Panel D, respectively. Additionally, $P_{\min }<P_{A}<P_{K}$ and these three values are respectively defined as follows.

$$
\begin{gathered}
P_{\min } \in \underset{P}{\arg } \int_{K}^{A}(x-K) f(x) d x-\bar{I}=0, \quad K=v+\bar{x}-\bar{I}, \bar{I}=C^{-1}(P) . \\
P_{A} \in \underset{P}{\arg } \int_{K}^{A}(x-K) f(x) d x+\int_{A}^{T}(x-A) f(x) d x-\bar{I}=0, \\
K=v+\bar{x}-\bar{I}, \bar{I}=C^{-1}(P) . \\
P_{K} \in \underset{P}{\arg } \int_{K}^{T}(x-K) f(x) d x-\bar{I}=0, \quad K=v+\bar{x}-\bar{I}, \bar{I}=C^{-1}(P) .
\end{gathered}
$$

Based on Definition 2, Panels A, B, C, and D in Fig. 2 display the double deductible insurance with a common threshold $A$. Additionally, expressions (10) and (11) are referred to Panels A and $\mathrm{C}$ respectively. And, $P_{K}$ denotes the insurance premium for the deductible insurance with a deductible $K$. Moreover, the following lemma shows that $P_{\min }, P_{A}$, and $P_{K}$ are uniquely determined.

Lemma 1. Each solution of expressions (10), (11), and (12) is unique; that is, $P_{\min }$, $P_{A}$, and $P_{K}$ are uniquely determined.

Proof: See Appendix 2.

Theorem 3. If $P<P_{\min }$, then no available insurance meets the VaR constraint. If $P \geq P_{K}$, then the optimal insurance is a single deductible insurance. Otherwise, if $P_{\min } \leq P<P_{K}$, then the optimal form of insurance is a double deductible insurance with a threshold A. Additionally, when the double deductible insurance is optimal, then the optimal retained loss schedule is as follows.

$$
R^{*}(x)=\left\{\begin{array}{ll}
\min \{x, K\} & \text { for } 0 \leq x \leq A \\
\min \left\{x, \bar{D}^{*}\right\} & \text { for } A<x \leq T
\end{array} \quad \text { if } P_{\min } \leq P<P_{K}\right.
$$




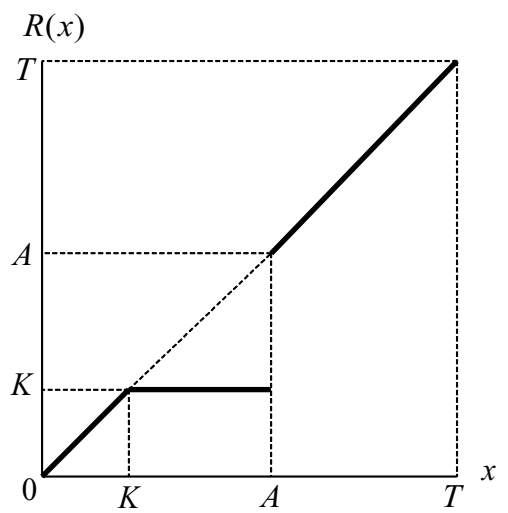

Panel A: $P=P_{\min }$

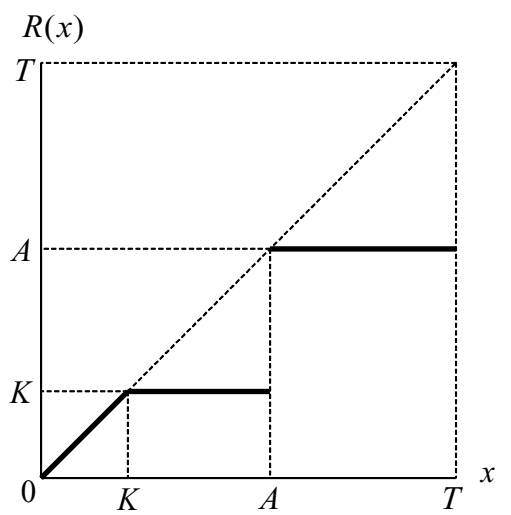

Panel C: $P=P_{A}$

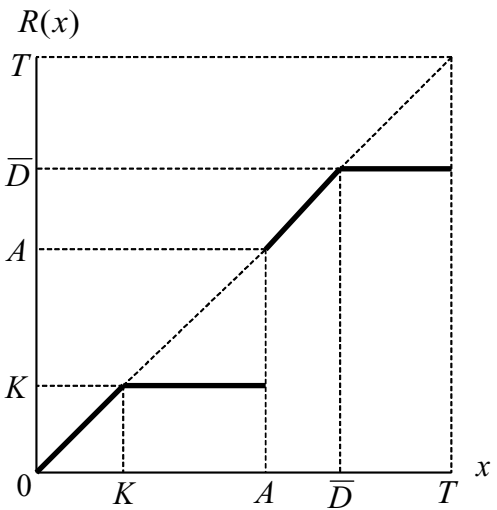

Panel B: $P_{\min }<P<P_{A}$

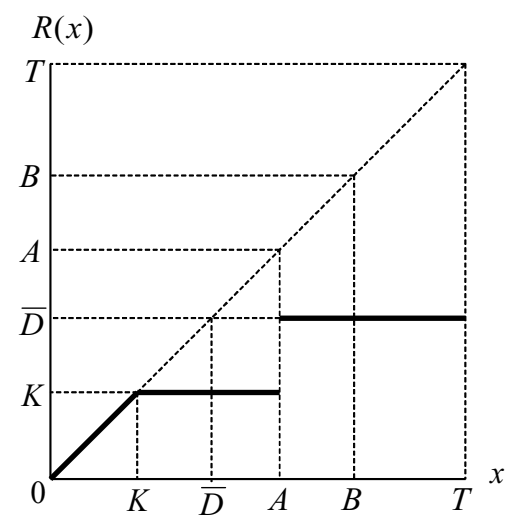

Panel D: $P_{A}<P<P_{K}$

Fig. 2 Double deductible insurance

where the threshold and the lower deductible exactly equals $A$ and $K$ respectively, and $\bar{D}^{*}$ represents the upper deductible such that

$$
\int_{0}^{A} \min \{x, K\} f(x) d x+\int_{A}^{T} \min \left\{x, \bar{D}^{*}\right\} f(x) d x=\bar{x}-C^{-1}(P)
$$

Proof: See Appendix 3.

The deductible for a single deductible insurance does not exceed $K$ when $P \geq P_{K}$ and thus the VaR constraint is redundant. Consequently, by Theorem 1, the single 
Table 1 Insurance premium and optimal contractual form*

\begin{tabular}{llll}
\hline$P$ & $P_{\min } \leq P \leq P_{A}$ & $P_{A}<P<P_{K}$ & $P_{K} \leq P$ \\
\hline $\begin{array}{l}\text { Insurance } \\
\text { form }\end{array}$ & Double deductible insurance & Double deductible insurance & Single deductible insurance \\
$R_{P}(x)$ & $\left\{\begin{array}{lll}\min \{x, K\} & \text { for } 0 \leq x \leq A \\
\min \{x, \bar{D}\} & \text { for } A<x \leq T\end{array}\right.$ & $\left\{\begin{array}{lll}\min \{x, K\} & \text { for } 0 \leq x \leq A & \min \left\{x, D^{*}\right\} \text { for } 0 \leq x \leq T \\
\bar{D} & \text { for } A<x \leq T & \\
\hline\end{array}\right.$
\end{tabular}

* The single deductible insurance is based on Theorem 1 and the double deductible insurance is based on Theorem 3. $A=F^{-1}(1-\alpha), K=\bar{x}+v-C^{-1}(P), P=C(\bar{I}) . A \leq \bar{D} \leq T$ for $P_{\min } \leq P \leq P_{A}$, and $K<\bar{D}<A$ for $P_{A}<P<P_{K}$. $D^{*}$ and $\bar{D}$ are solved from the equation: $E\left[R_{P}(X)\right]=\bar{x}-\bar{I}=\bar{x}-$ $C^{-1}(P) \cdot P_{\min }, P_{A}$, and $P_{K}$ are defined by expressions (10), (11), and (12), respectively. If $P<P_{\min }$ then no insurance contract can meet the VaR constraint; that is, no insurance contract is available.

deductible insurance is optimal. Particularly, when $P=P_{\min }$, the optimal insurance is the same as in Wang et al. [2005].

\section{Selections about insurance premium and contractual form}

This study divides the optimal insurance problem into two parts. Section 3 analyzed the first part, find the optimal $R(x)$ under fixed $P$. This section discusses the second part, determine the optimal $P$. The preceding discussion demonstrates that $R(x)$ only depends on $P$ for all increasing and concave utility functions. Restated, $R(x)$ can immediately be determined if $P$ is given, regardless of the preference of the insured. However, this result cannot indicate that $R(x)$ is not related to the utility function $U(\cdot)$. Indeed, $R(x)$ is a function of $P$ and $P$ is a function of $U(\cdot)$.

Let $R_{P}(x)$ denote the particular $R(x)$ corresponding to a specific $P$. Incorporating the results of Theorems 1 and $3, R_{P}(x)$ are listed in Table 1 . Meanwhile, $\bar{D} \geq A$ for $P_{\min } \leq P \leq P_{A}$ and $\bar{D}<A$ for $P_{A}<P<P_{K}$. Additionally, the optimality problem of expression (3) can be restated as follows.

$$
\underset{P \geq P_{\min }}{\operatorname{Maximize}} E[U(W)]=E\left[U\left(W_{0}-P-R_{P}(X)\right)\right]
$$

subject to $R_{P}(x)$ is defined by Table 1

Since if $P<P_{\min }$ then no insurance contract can meet the VaR constraint, $P \geq P_{\min }$ is a feasible premium. Without further specifying the distribution of $X$ and the utility function $U(\cdot)$, it is difficult to explicitly solve $P$. Fortunately, the numerical solution is easy to find. For instance, we can first select a sufficiently large premium, $P_{\max }$. Various premiums on interval $\left[P_{\min }, P_{\max }\right]$ are then included in expression (15) and an optimal $P^{*}$ which maximizes expression (15) is selected.

\footnotetext{
${ }^{4}$ The problem of Wang et al. [2005] is to maximize $E[W]$ subject to the VaR constraint. Since $E[W]$ is decreasing in $P$ owing to the loading fee, the optimal premium is the minimum premium $P_{\min }$ that just enough meets the VaR criterion.
} 


\section{Deductible insurance, upper-limit insurance, and proportional coinsurance}

In practice $I(x)$ is often limited in certain contractual forms, such as deductible insurance, upper-limit insurance, and proportional coinsurance, although these restrictions are unlikely to guarantee a Pareto optimum. Accordingly, with reference to the above methodology, this section demonstrates how the optimal coverage level is determined in these cases.

\section{Example 1: Deductible insurance}

Assume the indemnity schedule is limited to the form $I(x)=\max \{x-D, 0\}$, or equivalently $R(x)=\min \{x, D\}$, where $D$ denotes the deductible. Accordingly, the optimality problem is revised as follows.

$$
\underset{0 \leq D \leq T}{\operatorname{Maximize}} E[U(W)]=E\left[U\left(W_{0}-P-\min \{X, D\}\right)\right]
$$

subject to

$$
\begin{aligned}
P= & C(\bar{I}), \quad C(0)=0, \quad C^{\prime}(\bar{I})>1, \quad C^{\prime \prime}(\bar{I})>0 \\
\bar{I}= & E[I(X)]=E[X-\min \{X, D\}]=\int_{D}^{T}(x-D) f(x) d x \\
& \operatorname{Pr}\{\min \{X, D\} \leq K\} \geq 1-\alpha, \quad K \equiv v+\bar{x}-\bar{I}
\end{aligned}
$$

Expression (16c) implies that $\operatorname{Pr}\{\min \{X, D\} \leq K\}=1 \geq 1-\alpha$ if $D \leq K$ and $\operatorname{Pr}\{X \leq K\} \geq 1-\alpha$ if $D>K$. But $\operatorname{Pr}\{X \leq K\} \geq 1-\alpha$ implies $K \geq F^{-1}(1-$ $\alpha)=A$ and hence violates expression (4). Thus expression (16c) is equivalent to the relation $D \leq K$. Additionally, $D \leq K$ means that

$$
H_{d}(D) \equiv v+\bar{x}-\int_{D}^{T}(x-D) f(x) d x-D \geq 0
$$

From expression (17),

$$
H_{d}(T)=v+\bar{x}-T<0, H_{d}(0)=v>0, H_{d}^{\prime}(D)=-F(D)<0
$$

Expression (18) implies that a unique critical point $\bar{D}$ is contained in interval $[0, T]$ such that $H_{d}(\bar{D})=0$. Restated,

$$
\bar{D}=\underset{D}{\arg } H_{d}(D) \equiv v+\bar{x}-\int_{D}^{T}(x-D) f(x) d x-D=0
$$

Consequently, the optimality problem of expression (16) can be revised as follows.

$$
\underset{0 \leq D \leq \bar{D}}{\operatorname{Maximize}} E[U(W)]=E\left[U\left(W_{0}-C(\bar{I})-\min \{X, D\}\right)\right]
$$


with

$$
\bar{I}=\int_{D}^{T}(x-D) f(x) d x
$$

In this situation the optimality problem is that the insured selects the optimal deductible from interval $[0, \bar{D}]$ to maximize expression (20). Generally, the solution can be obtained using the fundamental calculus or the numerical method in the absence of an explicit solution.

\section{Example 2: Upper-limit insurance}

Assume the indemnity schedule is limited to $I(x)=\min \{x, M\}$ or equivalently $R(x)=\max \{0, x-M\}$, where $M$ denote the upper-limit. Accordingly, the optimality problem is revised as follows.

$$
\begin{aligned}
& \underset{0 \leq M \leq T}{\operatorname{Maximize}} E[U(W)]=E\left[U\left(W_{0}-P-\max \{0, X-M\}\right)\right] \\
& \text { subject to } \\
& P=C(\bar{I}), \quad C(0)=0, C^{\prime}(\bar{I})>1, C^{\prime \prime}(\bar{I})>0 \\
& \bar{I}=E[I(X)]=E[\min \{X, M\}]=\int_{0}^{M} x f(x) d x+M \int_{M}^{T} f(x) d x \\
& \\
& \quad \operatorname{Pr}\{\max \{0, X-M\} \leq K\} \geq 1-\alpha, \quad K \equiv v+\bar{x}-\bar{I}
\end{aligned}
$$

Since the relation $\max \{0, X-M\} \leq K$ clearly equals $X \leq K+M$, expression (21c) is the same as $K+M \geq F^{-1}(1-\alpha)=A$. Additionally, $K+M \geq A$ means that

$$
H_{u}(M) \equiv v+\bar{x}-\int_{0}^{M} x f(x) d x-M \int_{M}^{T} f(x) d x+M-A \geq 0
$$

From expression (22),

$$
H_{u}(0)=v+\bar{x}-A<0, H_{u}(T)=v+T-A>0, \text { and } H_{u}^{\prime}(M)=F(M)>0
$$

Expression (23) implies that a unique critical point $\underline{M}$ can be identified in interval $[0, T]$ such that $H_{u}(\underline{M})=0$. Restated,

$$
\underline{M}=\underset{\underline{M}}{\arg } H_{u}(M) \equiv v+\bar{x}-\int_{0}^{M} x f(x) d x-M \int_{M}^{T} f(x) d x+M-A=0
$$

Consequently, the optimality problem of expression (21) can be revised as follows.

$$
\underset{\underline{M} \leq M \leq T}{\operatorname{Maximize}} E[U(W)]=E\left[U\left(W_{0}-C(\bar{I})-\max \{0, X-M\}\right)\right]
$$


with

$$
\bar{I}=\int_{0}^{M} x f(x) d x+M \int_{M}^{T} f(x) d x
$$

Based on the similar approach in the deductible insurance, the insured would select the optimal upper-limit from interval $[\underline{M}, T]$ to maximize expression (25).

\section{Example 3: Proportional coinsurance}

Assume that the indemnity schedule is limited to proportional coinsurance, $I(x)=$ $\theta x$ or equivalently $R(x)=(1-\theta) x$, where $\theta$ represents the coinsurance proportion. Accordingly, the optimality problem is revised as follows.

$$
\begin{aligned}
& \underset{0 \leq \theta \leq 1}{\operatorname{Maximize}} E[U(W)]=E\left[U\left(W_{0}-P-(1-\theta) X\right)\right] \\
& \text { subject to } \\
& P=C(\bar{I}), \quad C(0)=0, C^{\prime}(\bar{I})>1, \quad C^{\prime \prime}(\bar{I})>0 \\
& \bar{I}=E[I(X)]=E[\theta X]=\theta \bar{x} \\
& \qquad \operatorname{Pr}\{(1-\theta) X \leq K\} \geq 1-\alpha, \quad K \equiv v+\bar{x}-\bar{I}
\end{aligned}
$$

Expression (26c) implied that

$$
\theta \geq 1-v /[A-\bar{x}]=\underline{\theta}
$$

where $0<\underline{\theta}<1$ since $A>v+\bar{x}$. Consequently, the optimality problem of expression (26) can be revised as follows.

$$
\underset{\underline{\theta} \leq \theta \leq 1}{\operatorname{Maximize}} E[U(W)]=E\left[U\left(W_{0}-C(\theta \bar{x})-(1-\theta) X\right)\right]
$$

Defining $J(\theta)=d E[U(W)] / d \theta$, then

$$
\begin{aligned}
J(\theta)= & E\left[\left(X-\bar{x} C^{\prime}(\theta \bar{x})\right) U^{\prime}\left(W_{0}-C(\theta \bar{x})-(1-\theta) X\right)\right] \\
J^{\prime}(\theta)= & E\left[\left(X-\bar{x} C^{\prime}(\theta \bar{x})\right)^{2} U^{\prime \prime}\left(W_{0}-C(\theta \bar{x})-(1-\theta) X\right)\right] \\
& +E\left[-\bar{x}^{2} C^{\prime \prime}(\theta \bar{x}) U^{\prime}\left(W_{0}-C(\theta \bar{x})-(1-\theta) X\right)\right]<0
\end{aligned}
$$

Expression (30) indicates that $E[U(W)]$ is globally concave with respect to $\theta$. Accordingly, the optimal coinsurance proportion: $\theta^{*}=\underline{\theta}$ if $J(\underline{\theta}) \leq 0$ and $\theta^{*}=1$ if $J(1) \geq 0$, otherwise $J\left(\theta^{*}\right)=0$. However, $J(1)<0$ since $C^{\prime}(\cdot)>1$. Consequently, $\theta^{*}$ is as follows.

$$
\begin{cases}\theta^{*}=\underline{\theta}=1-v /(A-\bar{x}) & \text { if } J(\underline{\theta}) \leq 0 \\ J\left(\theta^{*}\right)=0 & \text { otherwise }\end{cases}
$$


where $\underline{\theta}$ represents the corner solution and the inner solution satisfies $J\left(\theta^{*}\right)=0$. The proportional coinsurance can obtain a more explicit result rather than in the deductible insurance and the upper-limit insurance cases.

In sum, if the contractual form is given, then the optimal coverage levels (deductible size, upper-limit size, and coinsurance proportion) can be uniquely determined. Additionally, the solution process only involves one stage and frequently can be solved by fundamental calculus rather than variations of calculus.

\section{Conclusion}

This study aims to develop an optimal insurance contract endogenously under a VaR constraint, where the contract is obtained by maximizing the expected utility of the insured. Although Wang et al. [2005] had examined a similar topic, their assumptions implied that the insured was risk neutral. Consequently, this study extends Wang et al. [2005] and further considers a more realistic situation in which the insured is risk averse. The result demonstrates that the optimal insurance contract is a single deductible insurance when the VaR constraint is redundant or a double deductible insurance when the VaR constraint is binding.

To derive the above result, this study additionally obtains a preliminary theorem: when a risk has been insured against a specific loss interval, the optimal supplementary insurance also becomes a single deductible insurance for covering the remaining loss interval. Finally, the study specifically discusses the optimal coverage from the commonly existing forms of insurance, including deductible insurance, upper-limit insurance, and proportional coinsurance.

\section{Appendix 1: Proof of Theorem 2}

The optimal retained loss schedule is claimed to be

$$
R^{*}(x)= \begin{cases}R_{1}^{c}(x) & \text { for } x \in S_{1} \\ R_{2}^{*}(x) & \text { for } x \in S_{2}\end{cases}
$$

This means that $R^{*}(x)$ is not dominated by any $R(x)$, where $E\left[R^{*}(X)\right]=E[R(X)]$ and thus their premiums are equivalent based on expression (1). Mathematically,

$$
E\left[U\left(W_{0}-P-R^{*}(X)\right)\right] \geq E\left[U\left(W_{0}-P-R(X)\right)\right]
$$

From expressions (7), (8), and (A1), expression (A2) is expressed as

$$
\begin{aligned}
& \int_{S_{1}} U\left(W_{0}-P-R_{1}^{c}(x)\right) f(x) d x+\int_{S_{2}} U\left(W_{0}-P-R_{2}^{*}(x)\right) f(x) d x \\
& \quad \geq \int_{S_{1}} U\left(W_{0}-P-R_{1}^{c}(x)\right) f(x) d x+\int_{S_{2}} U\left(W_{0}-P-R_{2}(x)\right) f(x) d x
\end{aligned}
$$


Simplifying expression (A3) yields

$$
\int_{S_{2}} U\left(W_{0}-P-R_{2}^{*}(x)\right) f(x) d x \geq \int_{S_{2}} U\left(W_{0}-P-R_{2}(x)\right) f(x) d x
$$

Since $U^{\prime \prime}(\cdot)<0$, then

$$
\begin{aligned}
& U\left(W_{0}-P-R_{2}(x)\right)-U\left(W_{0}-P-R_{2}^{*}(x)\right) \\
& \quad \leq\left[R_{2}^{*}(x)-R_{2}(x)\right] U^{\prime}\left(W_{0}-P-R_{2}^{*}(x)\right) \text { for } x \in S_{2}
\end{aligned}
$$

It can be proved that

$$
\left[R_{2}^{*}(x)-R_{2}(x)\right] U^{\prime}\left(W_{0}-P-R_{2}^{*}(x)\right) \leq\left[R_{2}^{*}(x)-R_{2}(x)\right] U^{\prime}\left(W_{0}-P-D^{*}\right)
$$

The two cases $x \geq D^{*}$ and $0 \leq x<D^{*}$ are considered separately. If $x \geq D^{*}$, then $R_{2}^{*}(x)=D^{*}$ and hence

$$
\left[R_{2}^{*}(x)-R_{2}(x)\right] U^{\prime}\left(W_{0}-P-R_{2}^{*}(x)\right)=\left[R_{2}^{*}(x)-R_{2}(x)\right] U^{\prime}\left(W_{0}-P-D^{*}\right)
$$

If $0 \leq x<D^{*}$, then $R_{2}^{*}(x)=x<D^{*}$ and hence

$$
U^{\prime}\left(W_{0}-P-R_{2}^{*}(x)\right)<U^{\prime}\left(W_{0}-P-D^{*}\right)
$$

Since $0 \leq R_{2}(x) \leq x$, then

$$
R_{2}^{*}(x)-R_{2}(x) \geq 0
$$

Incorporating expressions (A8) with (A9) yields

$$
\left[R_{2}^{*}(x)-R_{2}(x)\right] U^{\prime}\left(W_{0}-P-R_{2}^{*}(x)\right) \leq\left[R_{2}^{*}(x)-R_{2}(x)\right] U^{\prime}\left(W_{0}-P-D^{*}\right)
$$

Expression (A6) has been proved based on expressions (A7) and (A10). Based on expressions (A5) and (A6), the following can be obtained.

$$
\begin{gathered}
\int_{S_{2}}\left\{U\left(W_{0}-P-R_{2}(x)\right)-U\left(W_{0}-P-R_{2}^{*}(x)\right)\right\} f(x) d x \\
\leq U^{\prime}\left(W_{0}-P-D^{*}\right) \int_{S_{2}}\left[R_{2}^{*}(x)-R_{2}(x)\right] f(x) d x
\end{gathered}
$$

Using equality $E\left[R^{*}(X)\right]=E[R(X)]=\bar{R}$ yields

$$
\int_{S_{1}} R_{1}^{c}(x) f(x) d x+\int_{S_{2}} R_{2}^{*}(x) f(x) d x=\int_{S_{1}} R_{1}^{c}(x) f(x) d x+\int_{S_{2}} R_{2}(x) f(x) d x
$$


Simplifying expression (A12) yields

$$
\int_{S_{2}}\left[R_{2}^{*}(x)-R_{2}(x)\right] f(x) d x=0
$$

Substituting expression (A13) into expression (A11) obtains the result of expression (A4). Expression (A4) equals expression (A2) and thus the proof is performed.

\section{Appendix 2: Proof of Lemma 1}

$G_{\min }(P), G_{A}(P)$, and $G_{K}(P)$ are respectively defined as follows.

$$
\begin{aligned}
G_{\min }(P) \equiv & \int_{K}^{A}(x-K) f(x) d x-\bar{I}, \quad K=v+\bar{x}-\bar{I}, \bar{I}=C^{-1}(P) . \\
G_{A}(P) \equiv & \int_{K}^{A}(x-K) f(x) d x+\int_{A}^{T}(x-A) f(x) d x-\bar{I}, \\
& K=v+\bar{x}-\bar{I}, \bar{I}=C^{-1}(P) . \\
G_{K}(P) \equiv & \int_{K}^{T}(x-K) f(x) d x-\bar{I}, \quad K=v+\bar{x}-\bar{I}, \bar{I}=C^{-1}(P) .
\end{aligned}
$$

From expression (1), $\bar{I}=0$ if $P=0$. Hence,

$$
G_{\min }(0)>0, G_{A}(0)>0 \text {, and } G_{K}(0)>0 \text {. }
$$

Respectively differentiating both sides of expressions (A14), (A15), and (A16) with respect to $P$ obtains

$$
\begin{aligned}
\frac{d G_{\min }(P)}{d P} & =\frac{d G_{A}(P)}{d P}=-\frac{d K}{d P}(K-K) f(K)+\int_{K}^{A}-\frac{d K}{d P} f(x) d x-\frac{d \bar{I}}{d P} \\
\frac{d G_{K}(P)}{d P} & =-\frac{d K}{d P}(K-K) f(K)+\int_{K}^{T}-\frac{d K}{d P} f(x) d x-\frac{d \bar{I}}{d P}
\end{aligned}
$$

Because of $d \bar{I} / d P>0$ by expression (1) and $d K / d P=-d \bar{I} / d P$ since $K=v+$ $\bar{x}-\bar{I}$, expressions (A18) and (A19) respectively imply that

$$
\begin{aligned}
d G_{\min }(P) / d P & =d G_{A}(P) / d P=-(d \bar{I} / d P)\{1-[F(A)-F(K)]\}<0 \\
d G_{K}(P) / d P & =-(d \bar{I} / d P)\{1-[1-F(K)]\}<0
\end{aligned}
$$

Expressions (A20) and (A21) mean that $G_{\min }, G_{A}$, and $G_{K}$ are always decreasing in $P$. Combining this fact with expression (A17) demonstrates each solution of expressions (10), (11), and (12) to be positive and unique. 


\section{Appendix 3: Proof of Theorem 3}

Lemma 1 have demonstrated that $P_{\min }, P_{A}$, and $P_{K}$ are unique, and thus they can be represented as critical points. For convenience, expression (13) is restated below.

$$
\begin{aligned}
& R^{*}(x)=\left\{\begin{array}{ll}
\min \{x, K\} & \text { for } 0 \leq x \leq A \\
\min \{x, \bar{D}\} & \text { for } A<x \leq T
\end{array} \text { if } P_{\min } \leq P \leq P_{A}\right. \\
& R^{*}(x)=\left\{\begin{array}{ll}
\min \{x, K\} & \text { for } 0 \leq x \leq A \\
\bar{D} & \text { for } A<x \leq T
\end{array} \text { if } P_{A}<P<P_{K}\right.
\end{aligned}
$$

where $A \leq \bar{D} \leq T$ in expression (A22) and $K<\bar{D}<A$ in expression (A23). The proof is based on the following four claims.

Claim 1. No insurance can meet the VaR constraint if $P<P_{\min }$.

If $P<P_{\min }$, then the retained loss on interval [K,A], shown in Panel A of Fig. 2, must increase for some loss $x$. However, this change deviates the VaR constraint of expression (3c) that $\operatorname{Pr}\{R(X) \leq K\} \geq 1-\alpha$. Consequently, Claim 1 is proven.

Claim 2. The optimal insurance is a single deductible insurance if $P \geq P_{K}$.

By definition, $P_{K}$ represents the premium for a deductible insurance with deductible $K$. Additionally, if $P \geq P_{K}$, then a deductible insurance can be found with a deductible not exceeding $K$ and which meets the VaR constraint. Claim 2 is proven by incorporating this fact with Theorem 1.

Claim 3. The optimal insurance is a double deductible insurance with a threshold $A$ if $P_{\min } \leq P \leq P_{A}$, and the optimal retained loss schedule is expression (A22).

For all $R(x), S$ can be partitioned into two subspaces, $S_{1}$ and $S_{2}$, such that

$$
S_{1}=\{x \mid 0 \leq R(x) \leq \hat{x}\}, \operatorname{Pr}\left\{S_{1}\right\}=1-\alpha ; S_{2}=\{x \mid \hat{x}<R(x) \leq T\}, \operatorname{Pr}\left\{S_{2}\right\}=\alpha .
$$

To meet the VaR constraint, the critical point $\hat{x} \leq K$ for all feasible retained loss schedules. Theorem 2 implies that the optimal insurance for the above partition of $S$ has the form (see Fig. 2 and Panel A of Fig. 3):

$$
R(x)=\left\{\begin{array}{lll}
\min \{x, \underline{D}\}, & 0<\underline{D} \leq K & \text { for } x \in S_{1} \\
\min \{x, \bar{D}\}, & A \leq \bar{D} \leq T & \text { for } x \in S_{2}
\end{array}\right.
$$

where $\underline{D}$ and $\bar{D}$ represent the lower and upper deductibles respectively. For $R^{*}(x)$ in expression (13), the corresponding $S_{1}, S_{2}, \underline{D}$, and $\bar{D}$ are as follows.

$$
S_{1}^{*}=\{x \mid 0 \leq x \leq A\}, \quad S_{2}^{*}=\{x \mid A<x \leq T\}, \quad \underline{D}=K, \text { and } \bar{D}=\bar{D}^{*} .
$$




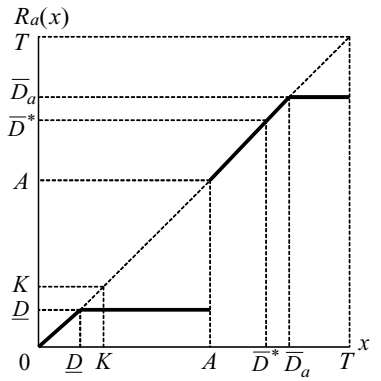

Panel A: $R_{a}(x)$

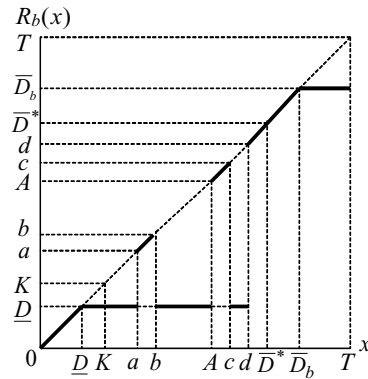

Panel B: $R_{b}(x)$

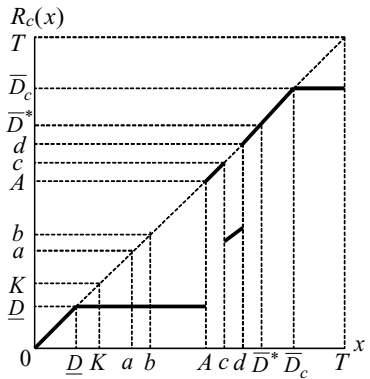

Panel C: $R_{c}(x)$

Fig. 3 Various feasible $R(x)$ for $P_{\min } \leq P \leq P_{A}$

By definition, the expected values of $R(X)$ and $R^{*}(X)$ are equivalent; restated,

$$
E[R(X)]=E\left[R^{*}(X)\right]
$$

This study will show $R^{*}(x)$ is not dominated by any $R(x)$ in expression (A25). Let $R_{a}(x), R_{b}(x)$, and $R_{c}(x)$ represent three various retained loss schedules, shown in Panels A, B, and C of Fig. 3. The three retained loss schedules have the same expected value and meet expressions (A24) and (A27). Meanwhile, $R_{a}(x)$ and $R_{b}(x)$ meet the specification of expression (A25). Moreover, let $\underline{D}_{a}=\underline{D}_{b}=\underline{D}_{c}=\underline{D}$ denote the lower deductible and $\bar{D}_{a}, \bar{D}_{b}, \bar{D}_{c}$ denote the upper deductibles. Furthermore, assume that $S_{1 a}, S_{1 b}, S_{1 c}$ and $S_{2 a}, S_{2 b}, S_{2 c}$ denote the respective $S_{1}$ and $S_{2}$ in expression (A24). Figure 3 demonstrates that

$$
S_{1 a}=S_{1 c}=S_{1}^{*}=\{x \mid 0 \leq x \leq A\}
$$

Actually, $R_{a}(x)$ is a representative retained loss schedule which is nondecresing in $x$; that is, $d R_{a}(x) / d x \geq 0$, shown in Panel A of Fig. 3. Since $E\left[R_{a}(X)\right]=E\left[R^{*}(X)\right]$ and $\underline{D} \leq K$, the relation $\bar{D}_{a} \geq \bar{D}^{*}$ is obtained. Additionally, $R_{b}(x)$ and $R_{c}(x)$ are viewed as representative retained loss schedules that do not require nondecasing in $x$, but $R_{c}(x)$ is further assumed that $S_{1 c}=\{x \mid 0 \leq x \leq A\}$, as shown in expression (A28). Although $R_{b}(x)$ is assumed that only two intervals ( $\{a \leq x \leq b\}$ and $\left.\{c \leq x \leq d\}\right)$ do not meet weakly monotonic. However, as the intervals (not monotonic) increase, the proof process is also the same. Accordingly, $R_{b}(x)$ can be represented a general form for expression (A25).

The proof of Claim 3 next is divided into three steps, as follows.

Step 1: $R_{c}(x)$ can be found for any $R_{b}(x)$ such that

$$
E\left[U\left(W_{0}-P-R_{b}(X)\right)\right]=E\left[U\left(W_{0}-P-R_{c}(X)\right)\right]
$$


First, the specific form of $R_{b}(x)$ shown on Panel B of Fig. 3 is considered. The corresponding $S_{1}$ and $S_{2}$ for $R_{b}(x)$ are as follows.

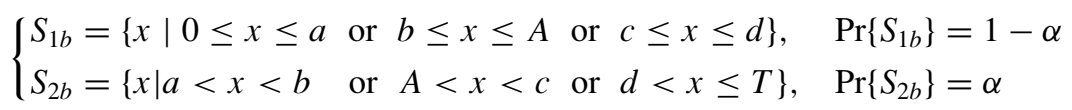

Furthermore, $R_{c}(x)$ shown as Panel C in Fig. 3 is found, such that

$$
\operatorname{Pr}\{a<X<b\}=\operatorname{Pr}\{c<X<d\}
$$

Let $a \leq R_{c}(x)=\beta_{0}+\beta_{1} x \leq b<x$ for $c \leq x \leq d$, then $\beta_{0}$ and $\beta_{1}$ can be found such that

$$
\int_{a}^{b} x f(x) d x=\int_{c}^{d}\left(\beta_{0}+\beta_{1} x\right) f(x) d x
$$

and

$$
\int_{a}^{b} U\left(W_{0}-P-x\right) f(x) d x=\int_{c}^{d} U\left(W_{0}-P-\beta_{0}-\beta_{1} x\right) f(x) d x
$$

must be equivalent for $R_{b}(x)$ and $R_{c}(x)$. Incorporating the fact with expression (A31), (A32), and (A33) can yield the following trivial result.

$$
E\left[U\left(W_{0}-P-R_{b}(X)\right)\right]=E\left[U\left(W_{0}-P-R_{c}(X)\right)\right]
$$

Although only the special case of $R_{b}(x)$ is provided here, the proof process demonstrates that any form of $R_{b}(x)$ can yield the same result. Accordingly, it can be found a $R_{c}(x)$ to satisfy expression (A29) for any $R_{b}(x)$.

Step 2: $R_{a}(x)$ is not dominated by $R_{c}(x)$. That is,

$$
E\left[U\left(W_{0}-P-R_{a}(X)\right)\right] \geq E\left[U\left(W_{0}-P-R_{c}(X)\right)\right]
$$

The general forms of $R_{a}(x)$ and $R_{c}(x)$ are as follows.

$$
\begin{aligned}
& R_{a}(x)=\left\{\begin{array}{lll}
\min \{x, \underline{D}\}, & 0<\underline{D} \leq K & \text { for } 0 \leq x \leq A \\
\min \left\{x, \bar{D}_{a}\right\}, & A \leq \bar{D}_{a} \leq T & \text { for } A<x \leq T
\end{array}\right. \\
& R_{c}(x)=\left\{\begin{array}{ll}
\min \{x, \underline{D}\}, & 0<\underline{D} \leq K \\
R_{c 2}(x)>\underline{D}, & \text { for } A<x \leq T
\end{array} \text { for } 0 \leq x \leq A\right.
\end{aligned}
$$

The notation $R_{c 2}(x)$ (corresponding to $S_{2 c}$ ) denotes that $R_{c}(x)$ has no specific form for $A<x \leq T$. Since $U^{\prime \prime}(\cdot)<0$, then

$$
\begin{aligned}
& U\left(W_{0}-P-R_{c}(x)\right)-U\left(W_{0}-P-R_{a}(x)\right) \\
& \quad \leq\left[R_{a}(x)-R_{c}(x)\right] U^{\prime}\left(W_{0}-P-R_{a}(x)\right) \text { for } 0 \leq x \leq T .
\end{aligned}
$$


Additionally, the following three cases are considered to demonstrate

$$
\begin{aligned}
& {\left[R_{a}(x)-R_{c}(x)\right] U^{\prime}\left(W_{0}-P-R_{a}(x)\right)} \\
& \quad \leq\left[R_{a}(x)-R_{c}(x)\right] U^{\prime}\left(W_{0}-P-\bar{D}_{a}\right) \text { for } 0 \leq x \leq T .
\end{aligned}
$$

Case 1: $0 \leq x \leq A$. In this case, $R_{a}(x)=R_{c}(x)$ and thus the left and right hand sides are equal for expression (A39).

Case 2: $A<x<\bar{D}_{a}$. In this case, $R_{c}(x) \leq R_{a}(x)=x<\bar{D}_{a}$. Since $U^{\prime \prime}(x)<0$, then $U^{\prime}\left(W_{0}-P-R_{a}(x)\right) \leq U^{\prime}\left(W_{0}-P-\bar{D}_{a}\right)$. Accordingly, the result of expression (A39) is obtained for $A<x<\bar{D}_{a}$.

Case 3: $\bar{D}_{a} \leq x \leq T$. In this case, $R_{a}(x)=\bar{D}_{a}$ and thus the left and right hand sides are equal for expression (A39).

The above three cases demonstrate expression (A39). Incorporating expressions (A38) with (A39) yields

$$
\begin{aligned}
& U\left(W_{0}-P-R_{c}(x)\right)-U\left(W_{0}-P-R_{a}(x)\right) \\
& \quad \leq\left[R_{a}(x)-R_{c}(x)\right] U^{\prime}\left(W_{0}-P-\bar{D}_{a}\right) \text { for } 0 \leq x \leq T .
\end{aligned}
$$

From expressions (A27) and (A40), then

$$
\begin{aligned}
E & {\left[U\left(W_{0}-P-R_{c}(X)\right)\right]-E\left[U\left(W_{0}-P-R_{a}(X)\right)\right] } \\
& =\int_{0}^{T}\left[U\left(W_{0}-P-R_{c}(x)\right)-U\left(W_{0}-P-R_{a}(x)\right)\right] f(x) d x \\
& \leq \int_{0}^{T} U^{\prime}\left(W_{0}-P-\bar{D}_{a}\right)\left[R_{a}(x)-R_{c}(x)\right] f(x) d x \\
& =U^{\prime}\left(W_{0}-P-\bar{D}_{a}\right)\left\{E\left[R_{a}(X)\right]-E\left[R_{c}(X)\right]\right\}=0
\end{aligned}
$$

From expression (A41), step 2 is completed.

Step 3: $R^{*}(x)$ is not dominated by $R_{a}(x)$. Restated,

$$
E\left[U\left(W_{0}-P-R^{*}(X)\right)\right] \geq E\left[U\left(W_{0}-P-R_{a}(X)\right)\right]
$$

Since $E\left[R_{a}(x)\right]=E\left[R^{*}(x)\right]$ and $\underline{D} \leq K$, then $\bar{D}_{a} \geq \bar{D}^{*}$. Since $U^{\prime \prime}(\cdot)<0$,

$$
\begin{aligned}
& U\left(W_{0}-P-R_{a}(x)\right)-U\left(W_{0}-P-R^{*}(x)\right) \\
& \quad \leq\left[R^{*}(x)-R_{a}(x)\right] U^{\prime}\left(W_{0}-P-R^{*}(x)\right) \text { for } 0 \leq x \leq T
\end{aligned}
$$

The two cases $0 \leq x \leq A$ and $A<x \leq T$ are considered separately to demonstrate

$$
\begin{aligned}
& {\left[R^{*}(x)-R_{a}(x)\right] U^{\prime}\left(W_{0}-P-R^{*}(x)\right)} \\
& \quad \leq\left[R^{*}(x)-R_{a}(x)\right] U^{\prime}\left(W_{0}-P-A\right) \text { for } 0 \leq x \leq T .
\end{aligned}
$$


Case 1: $0 \leq x \leq A$. In this case, we have $R_{a}(x) \leq R^{*}(x) \leq A$. Since $U^{\prime \prime}(x)<0$, $U^{\prime}\left(W_{0}-P-R^{*}(x)\right) \leq U^{\prime}\left(W_{0}-P-A\right)$. Accordingly, the result of expression (A44) is obtained for $0 \leq x \leq A$.

Case 2: $A<x \leq T$. In this case, $A \leq R^{*}(x) \leq R_{a}(x)$. Since $U^{\prime \prime}(x)<0$, the relation $U^{\prime}\left(W_{0}-P-R^{*}(x)\right) \geq U^{\prime}\left(W_{0}-P-A\right)$ is obtained. Accordingly, the result of expression (A56) is obtained for $A<x \leq T$.

From the above two cases, expression (A4) has been demonstrated. Incorporating expressions (A43) with (A44) yields

$$
\begin{aligned}
& U\left(W_{0}-P-R_{a}(x)\right)-U\left(W_{0}-P-R^{*}(x)\right) \\
& \quad \leq\left[R^{*}(x)-R_{a}(x)\right] U^{\prime}\left(W_{0}-P-A\right) \text { for } 0 \leq x \leq T .
\end{aligned}
$$

From expressions (A27) and (A45),

$$
\begin{aligned}
E & {\left[U\left(W_{0}-P-R_{a}(X)\right)\right]-E\left[U\left(W_{0}-P-R^{*}(X)\right)\right] } \\
& =\int_{0}^{T}\left[U\left(W_{0}-P-R_{a}(x)\right)-U\left(W_{0}-P-R^{*}(x)\right)\right] f(x) d x \\
& \leq \int_{0}^{T} U^{\prime}\left(W_{0}-P-A\right)\left[R^{*}(x)-R_{a}(x)\right] f(x) d x \\
& =U^{\prime}\left(W_{0}-P-A\right)\left\{E\left[R^{*}(X)\right]-E\left[R_{a}(X)\right]\right\}=0
\end{aligned}
$$

Expression (A42) is demonstrated based on expression (A46). Consequently, Claim 3 is demonstrated based on steps 1,2 , and 3 .

Claim 4: The optimal insurance is a double deductible insurance with a threshold $A$ if $P_{A}<P<P_{K}$, and the optimal retained loss schedule is expression (A23).

Using the same definitions for $R_{a}(x), R_{b}(x)$, and $R_{c}(x)$ in Claim 3 , as in the analysis presented in Claim 3, the same results can be obtained. First, $R_{c}(x)$ can be identified for any $R_{b}(x)$ such that $R_{c}(x)$ and $R_{b}(x)$ have the same expected utility. Second, $R_{c}(x)$ does not dominate $R_{a}(x)$. Accordingly, Claim 4 can be proven by further domonstrating that $R_{a}(x)$ does not dominate $R^{*}(x)$. Corresponding to expression (A23), the definition of $R_{a}(x)$ in expression (A36) must be slightly modified as follows.

$$
R_{a}(x)=\left\{\begin{array}{lll}
\min \{x, \underline{D}\}, & 0<\underline{D} \leq K & \text { for } 0 \leq x \leq A \\
\min \left\{x, \bar{D}_{a}\right\}, & K<\bar{D}_{a} \leq T & \text { for } A<x \leq T
\end{array}\right.
$$

Although Panel A graphs only the case $A<\bar{D}^{*}<\bar{D}_{a}$, the proof process is the same for the others: $\bar{D}^{*}<A<\bar{D}_{a}$ and $\bar{D}^{*}<\bar{D}_{a}<A$. Since $U^{\prime \prime}(\cdot)<0$, the same result of expression (A55) can be obtained. Additionally, $R_{a}(x) \leq R^{*}(x)<\bar{D}^{*}$ for $0 \leq x \leq A$, and $R^{*}(x)=\bar{D}^{*}$ for $A<x \leq T$. This implies

$$
\begin{aligned}
& {\left[R^{*}(x)-R_{a}(x)\right] U^{\prime}\left(W_{0}-P-R^{*}(x)\right)} \\
& \quad \leq\left[R^{*}(x)-R_{a}(x)\right] U^{\prime}\left(W_{0}-P-\bar{D}^{*}\right) \text { for } 0 \leq x \leq T .
\end{aligned}
$$


Like Claim 3, incorporating expressions (A43) with (A48) yields Claim 4.

\section{Summary}

From Claims 1, 2, 3, and 4, the proof of Theorem 3 has been completed.

Acknowledgments We are grateful to the editor and two referees for many helpful comments and suggestions.

\section{References}

BOWERS, N.L., GERBER, H.U., HICKMAN, J.C., JONES, D.A., and NESBITT, C.J. [1986]: Actuarial Mathematics, Society of Actuaries.

DOHERTY, N.A. and SCHLESINGER, H. [1983a]: “Optimal Insurance in Incomplete Markets,” Journal of Political Economy, 91, 1045-1054.

DOHERTY, N.A. and SCHLESINGER, H. [1983b]: "The Optimal Deductible for an Insurance Policy When Initial Wealth is Random," Journal of Business, 56, 555-565.

ERMOLIEV, Y.M. and FLAM, S.D. [2001]: "Finding Pareto Optimal Contracts," The Geneva Papers on Risk and Insurance Theory, 26, 155-167.

FRYER, M.J. and GREENMAN J.V. [1987]: Optimisation Theory: Applications in OR and Economics, Edwad Arnold Ltd.

GOLLIER, C. [1987]: "The Design of Optimal Insurance Contracts without the Nonnegativity Constraint on Claims," Journal of Risk and Insurance, 54, 314-324.

GOLLIER, C. [1996]: “Optimal Insurance of Approximate Losses,” Journal of Risk and Insurance, 63, 369-380.

HUBERMAN, G., MAYERS, D., and SMITH, C.W. [1983]: "Optimal Insurance Policy Indemnity Schedules," Bell Journal of Economics, 14, 415-426.

JORION, P. [2001]: Value at Risk: The New Benchmark for Managing Financial Risk, McGraw-Hill International Edition.

RAVIV, A. [1979]: “The Design of an Optimal Insurance Policy,” American Economic Review, 69, 84-96.

SCHLESINGER, H. [1981]: “The Optimal Level of Deductibility in Insurance Contracts," Journal of Risk and Insurance, 48, 465-481.

SPAETER, S. and ROGER, P. [1997]: “The Design of Optimal Insurance Contracts: A Topological Approach," The Geneva Papers on Risk and Insurance Theory, 22, 5-19.

WANG, C.P., SHYU, D., and HUANG, H.H. [2005]: “Optimal Insurance Design under a Value-at-Risk Framework," The Geneva Risk and Insurance Review, 30, 161-179. 